\title{
High Speed and High Precision Fs-laser Writing Using a Scanner with Large Numerical Aperture
}

\author{
Jens GOTTMANN, Maren HÖRSTMANN-JUNGEMANN, Martin HERMANS, Dennis BECKMANN \\ Lehrstuhl für Lasertechnik, RWTH Aachen University, Steinbachstraße 15, 52074 Aachen, Germany \\ E-mail: jens.gottmann@ilt.fraunhofer.de
}

\begin{abstract}
Currently available high speed scanning systems based on galvo mirrors are equipped with objectives with small numerical apertures $(\mathrm{NA}<0.2)$ which are not sufficient for most of the involume micro structuring processes. Whereas high precision air bearing translation stages are used with microscope objectives the speed is limited to approximately $100 \mathrm{~mm} / \mathrm{s}$. In order to overcome these limitations we integrated a galvo scanner into a system with large maximum scanning velocity $(50-400 \mathrm{~mm} / \mathrm{s})$, large numerical aperture $(\mathrm{NA}=0.4-1.2)$, small focus size $(0.6-2.2 \mu \mathrm{m})$, high precision $(100-400 \mathrm{~nm})$ and computer controlled pre-compensation of spherical aberrations for involume focusing. Furthermore, we combined the scanning system with a computer controlled three axis translation stage to process large flat work pieces such as wafers up to $2 \mathrm{~mm}$ in thickness. As application of the scanner high speed direct writing of waveguides, markings, nano structures inside transparent materials as well as the high speed fabrication of 3D micro structures by in-volume selective laser etching are demonstrated.

DOI: 10.2961/jlmn.2009.03.0009
\end{abstract}

Keywords: Scanning system, fs-laser direct writing, in-volume modification, fs-laser micro structuring, in-volume selective laser etching

\section{Introduction}

Micro structuring in the volume of transparent materials is enabled by ultrafast laser radiation. By laser radiation with pulse durations in the fs and ps regime multi photon processes are efficiently induced resulting in high resolution $<1 \mu \mathrm{m}^{3}$, very low heat input and high writing flexibility in three dimensions. High transparent materials such as glasses and sapphire are locally modified in-volume to change the refractive index for optical applications [1] or to selectively increase the corrodibility locally for the manufacturing of micro channels and micro structured parts by in-volume selective laser induced etching for use in micro system and medical technology [2, 3, 4].

Exploiting ultrafast laser radiation with repetition rates up to several $\mathrm{MHz}$ (e.g. from FCPA fs lasers) high manufacturing velocities are possible, if either the work piece or the laser beam could be moved fast enough [5].

Currently available high speed scanning systems based on galvo mirrors are equipped with objectives with small numerical apertures $(\mathrm{NA}<0.2)$. Therefore, the threshold for self focusing in the volume of transparent materials is reached before the threshold for dielectric breakdown, often resulting in the formation of cracks instead of the desired homogenous modification of the refractive index or the corrodibility [6]. Three axes micro positioning stages exhibit high precision and the laser radiation can be focused using microscope objectives with large numerical apertures. However, because large masses have to be accelerated, the dynamics of the motion is limited resulting in low productivity of producing 3D shaped micro structures. Therefore, to exploit the productivity of high repetition rate fs-lasers high dynamic galvo scanners have to be combined with microscope objectives with large numerical apertures for in-volume structuring. A simple combination of a galvo mirrors with microscope objectives results in very small scanning fields and distortion, because the exit pupil of the lens is typically several $\mathrm{mm}$ inside the objective. Therefore, we use a 1:1 telescope to image the galvo mirror into the exit pupil of the microscope objective.

\section{Pre-compensation of spherical aberrations}

A planar plate with a refractive index different from the air or the immersion liquid located between a focusing lens and the focal point results in spherical aberrations. Therefore, spherical aberrations must be pre-compensated during focusing in the volume of transparent materials at variable depth e.g. using the correction collar of microscope objectives. During focusing with numerical aperture $N A$ in the depth $d$ of a material with refractive index $n$ surrounded by a medium with refractive index $n_{0}$ the spherical aberration results in an increased focal diameter and an elongation of the length of the focus $L$ :

$$
L=d \frac{n_{0}}{n}\left(\sqrt{\frac{n^{2}-N A^{2}}{n_{0}^{2}-N A^{2}}}-n\right)
$$

Equation (1) is directly derived from simple geometrical considerations: The maximum angle of the incident rays is $\alpha$ in the surrounding medium and $\alpha^{\prime}$ in the medium and the numerical aperture is $\mathrm{NA}=n_{0} \sin \alpha=n \sin \alpha^{\prime}$. Following Snells law the refracted rays with the maximum angle $\alpha^{\prime}$ hit the optical axis in the glass at the deepest point $z_{\max }$ :

$$
z_{\max }=d \frac{n_{0}}{n}\left(\frac{\sin \alpha \cos \alpha^{\prime}}{\cos \alpha \sin \alpha^{\prime}}\right)=d \frac{n_{0}}{n}\left(\sqrt{\frac{n^{2}-N A^{2}}{n_{0}^{2}-N A^{2}}}\right)
$$

The elongation of the length of the focus $L$ is the difference between the deepest intersection with the optical axis 
$Z_{\max }$ and the intersection of rays with small angles with the optical axis at $d$, resulting directly in equation (1).

The spherical aberration becomes significant, if the elongation $L$ is increased to the value of the length of the focus without aberrations $2 z_{R}=2 \omega_{0} / N A, \omega_{0}=\lambda / \pi N A$. For example during focusing by a lens in air with a numerical aperture of $\mathrm{NA}=0.5$ inside glass the spherical aberration becomes significant for un-compensated glass thickness larger than $30 \mu \mathrm{m}$ (Figure 1). Because other nonlinear effects during the propagation of ultrafast laser radiation in transparent materials also result in an elongation of the length of the focus, the un-compensated glass thickness may be somewhat larger before effects of spherical aberrations become remarkable. Nevertheless, for 3D micro structuring in the volume of glass several $100 \mu \mathrm{m}$ in thickness with numerical apertures $>0.4$ the spherical aberrations have to be pre-compensated. Therefore, we have equipped the correction collar of corrected microscope objectives [7] with computer controlled motor to allow dynamic pre-compensation.

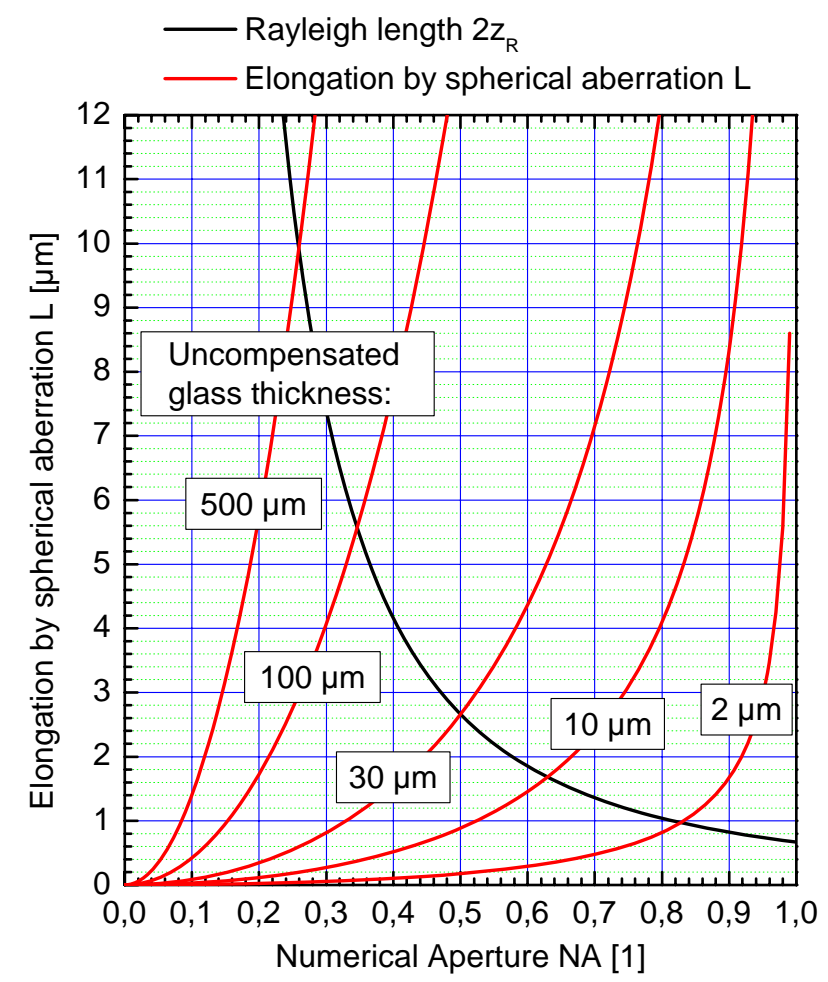

Figure 1: Elongation of length of focus L by spherical aberration due to a plane glass plate

\section{Scanner system and experimental}

A commercial galvo scanner (hurrySCAN 10 by ScanLab) is used for scanning. The galvo mirrors are imaged to the plane of the exit pupil of the telecentric microscope objective using a 1:1 telescope designed for minimal aberrations (Figure 2). The correction collar of the microscope objective is equipped with a pinion driven by a computer controlled motor. The integrated system (Figure 3 ) is combined with a three axes translation stage (microstep by Kugler) for stepping in three dimensions. The galvo mirrors, the correction collar and the translation stage are con- trolled by CAD/CAM software (modified versions of scan2D and scan3D by Fraunhofer ILT).

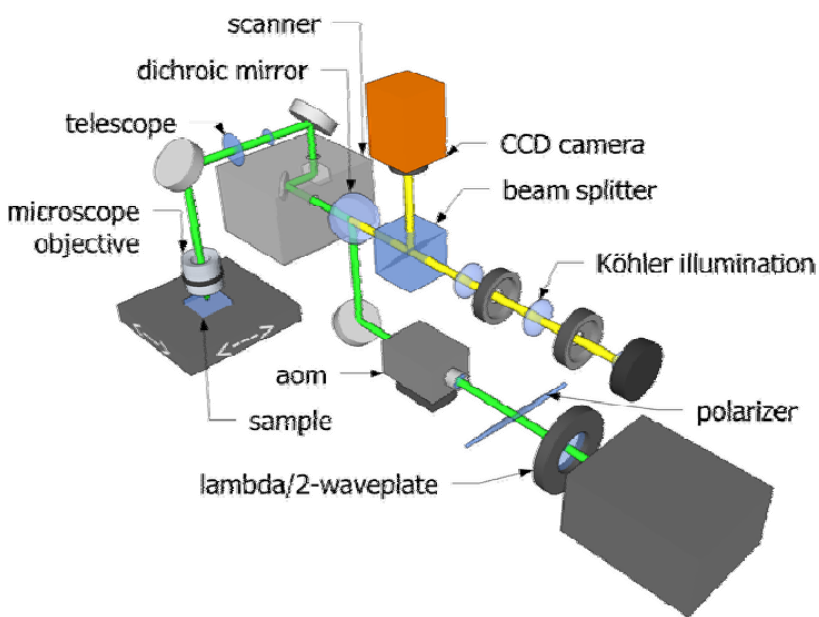

Figure 2: Optics scheme of the set-up, including the scanning system consisting of galvo mirrors, telescope and microscope objective

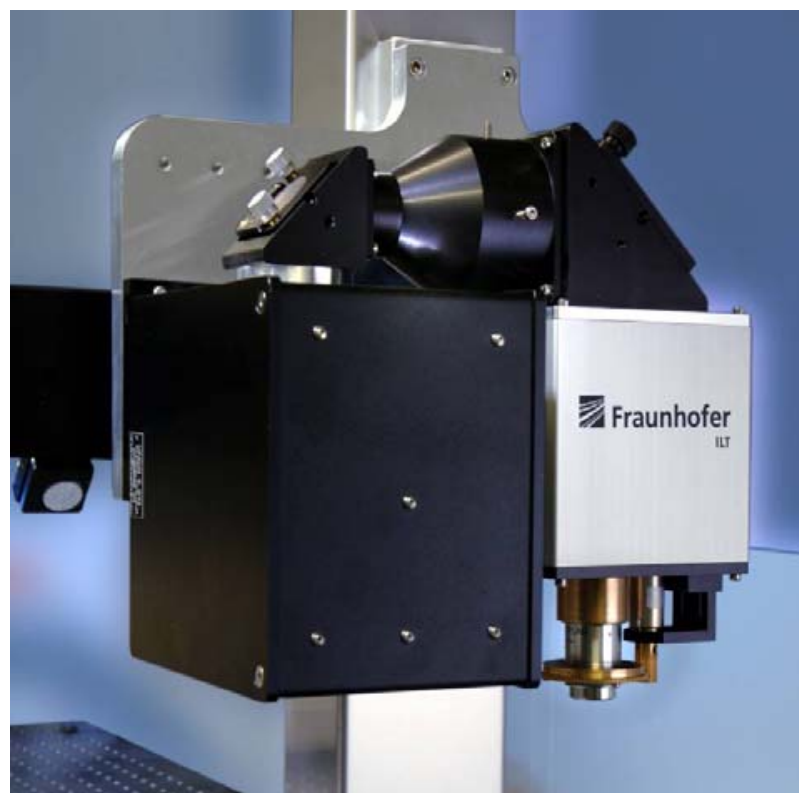

Figure 3: Scanning system consisting of galvo mirrors, telescope and microscope objective with a motorized correction collar for pre-compensation of spherical aberrations

Table 1 Parameters of the scanning system with some of the microscope objectives used

\begin{tabular}{ccccc}
\hline Objective & Focus $\varnothing$ Precision Mark & $\begin{array}{l}\text { Jump } \\
\text { velocity }\end{array}$ \\
& & & & \\
\hline $20 \mathrm{x} / 0.4$ & $1.66 \mu \mathrm{m}$ & $430 \mathrm{~nm}$ & $103 \mathrm{~mm} / \mathrm{s}$ & $360 \mathrm{~mm} / \mathrm{s}$ \\
$40 \mathrm{x} / 0.6$ & $1.11 \mu \mathrm{m}$ & $210 \mathrm{~nm}$ & $51 \mathrm{~mm} / \mathrm{s}$ & $180 \mathrm{~mm} / \mathrm{s}$ \\
$40 \mathrm{x} / 1.2 \mathrm{~W}$ & $0.55 \mu \mathrm{m}$ & $210 \mathrm{~nm}$ & $51 \mathrm{~mm} / \mathrm{s}$ & $180 \mathrm{~mm} / \mathrm{s}$ \\
$100 \mathrm{x} / 1.3 \mathrm{Oil}$ & $0.51 \mu \mathrm{m}$ & $86 \mathrm{~nm}$ & $21 \mathrm{~mm} / \mathrm{s}$ & $72 \mathrm{~mm} / \mathrm{s}$ \\
\hline
\end{tabular}

Depending on the numerical aperture and the magnification of the used microscope objective the focus diameter, the precision, the maximum constant velocity and the maximum jump velocity result (Table 1 ). The usable scan- 
ning field diameter is $25 \mathrm{~mm}$ divided by the magnification of the respective microscope objective.

The irradiation is carried out with an Yb-glass fiber chirped pulse amplifier (FCPA) IMRA $\mu$ Jewel with a central wavelength of $\lambda=1045 \mathrm{~nm}$, a pulse duration $\tau=450 \mathrm{fs}$ and a variable repetition rate $\mathrm{f}=100 \mathrm{kHz}-5 \mathrm{MHz}$. The maximum average power of $\mathrm{P}_{\mathrm{av}}=1.5 \mathrm{~W}$ is adjustable using a half wavelength retardation plate and a linear polarizer. The laser radiation is modulated by an acousto-optical modulator (PM 106 from IMRA) triggered by the scanner to gate the laser radiation on and off. The laser beam is transferred into the scanning system and focused by microscope objectives using a numerical aperture of 0.3 $(20 \mathrm{x} / 0.4)$ and $0.6(40 \mathrm{x} / 0.6)$. The sample is mounted on a programmable three axis translation stage (accuracy $\Delta x=$ $100 \mathrm{~nm}$, maximum speed $\mathrm{v}_{\max }=2 \mathrm{~mm} / \mathrm{s}$ ). The materials used are sapphire with the crystalline orientation of (0001), fused silica and boro-silicate glass (D263 from Schott) with a sample size of $10 \mathrm{~mm} \times 10 \mathrm{~mm} \times 1 \mathrm{~mm}$. Top and bottom surfaces are polished to optical quality. The irradiation is carried out in ambient air.

\section{Examples for high speed scanning}

\subsection{Waveguide writing}

Direct laser writing of waveguides in boro-silicate glass (D263 from Schott) is done using a microscope objective with numerical aperture of $\mathrm{NA}=0.6$, a repetition rate of 1 $\mathrm{MHz}$ and a pulse energy of $\mathrm{E}_{\mathrm{P}}=225 \mathrm{~nJ}$. The numerical aperture of the resulting waveguides is measured using far field measurements and the refractive index modification is analyzed using interference microscopy [8]. Straight lines are written with a scanning velocity of $100 \mathrm{~mm} / \mathrm{s}$ resulting in homogeneous crack-free waveguides (Figure 4, left). Curved waveguides with radii of $400-600 \mu \mathrm{m}$ are written using a scanning velocity of $50 \mathrm{~mm} / \mathrm{s}$. The curved waveguides exhibit in-homogeneities caused by the vectorization of the circles (Figure 4, right). To prevent the inhomogeneities the scanning software has to be modified to allow for constant velocity during scanning a curve and the development of an adapted processing strategy is necessary in future work.

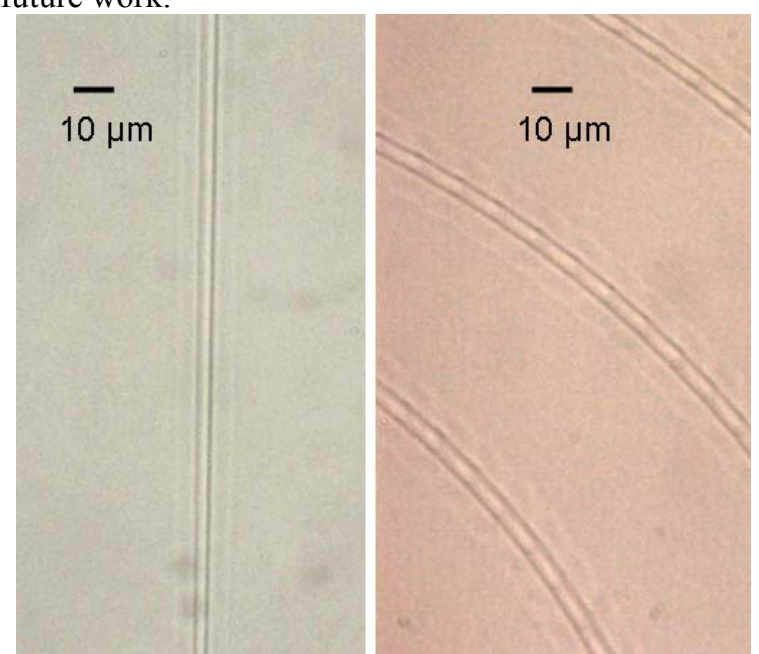

Figure 4: Straight (left) and curved waveguides (right) in D263 glass by direct fs-laser writing

\subsection{Markings inside transparent materials}

Micro markings in fused silica are produced by direct laser writing $200 \mu \mathrm{m}$ below the polished surface using an effective numerical aperture of $\mathrm{NA}=0.3$ (objective $20 \mathrm{x} / 0.4$, beam diameter $5 \mathrm{~mm}$ ). The spherical aberrations caused by $200 \mu \mathrm{m}$ glass are pre-compensated using the correction collar. Using a repetition rate of $500 \mathrm{kHz}$, an average power of $320 \mathrm{~mW}$ an a scanning velocity of $100 \mathrm{~mm} / \mathrm{s}$ a logo is written in less than 1 second (Figure 5). No distortion of the written grid is observed in the scanning field $1.3 \mathrm{~mm}$ in diameter (Figure 5).

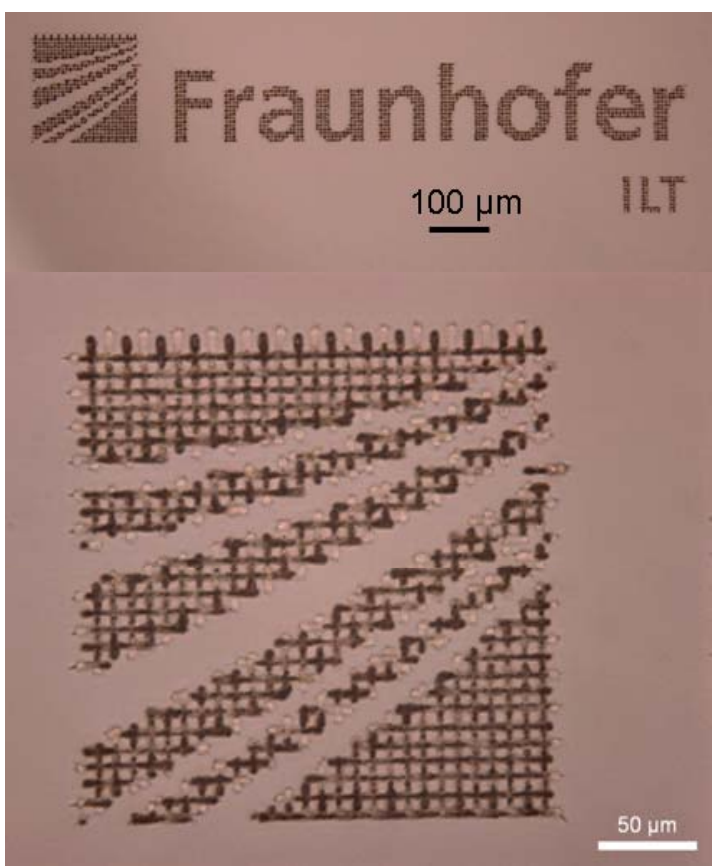

Figure 5: Marking inside fused silica by direct laser writing: Logo (top) and detail (bottom)
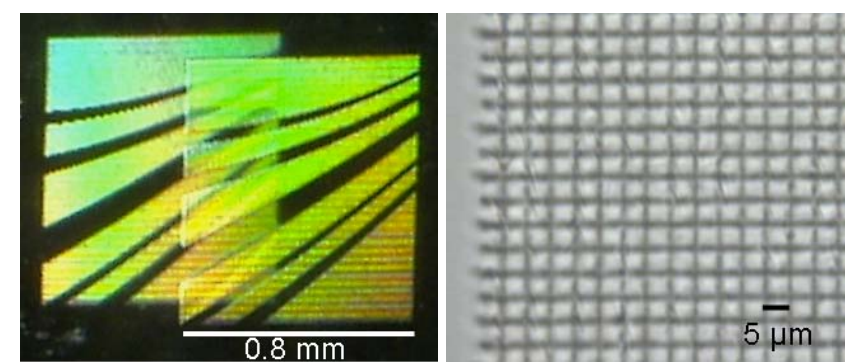

Figure 6: Colored marking inside sapphire by direct laser writing: Logo (left, color by diffraction) and detail (right)

Micro markings in sapphire are produced by direct laser writing $250 \mu \mathrm{m}$ below the polished surface using an effective numerical aperture of $\mathrm{NA}=0.3$. The correction collar for pre-compensation of the spherical aberrations is set to $290 \mathrm{~nm}$ to account for the different refractive index of sapphire. The repetition rate has been $500 \mathrm{kHz}$, the average power $320 \mathrm{~mW}$ and the scanning velocity $100 \mathrm{~mm} / \mathrm{s}$. A logo $0.8 \times 0.8 \mathrm{~mm}^{2}$ in size has been written consisting of parallel lines with an offset of $5 \mu \mathrm{m}$ in about 3 seconds (Figure 6). As measured by interference microscopy the modified lines exhibit a reduced refractive index $(\Delta n=-$ 0.03 ) and no cracks are observed (Figure 6 , right). Due to 
diffraction of light at the laser written micro grid and interference in the observers eye colors are observed varying with the angle (Figure 6, left).

\subsection{In-volume selective laser etching}

3D micro structures are written in sapphire $1 \mathrm{~mm}$ in thickness using the same parameters as for direct laser writing of markings in sapphire described above. Additionally the sample is moved in perpendicular to the beam propagation axes (z-scan) using the micro positioning stage. In this way structures $1 \mathrm{~mm}$ in diameter and $1 \mathrm{~mm}$ in height (from the bottom to the top of the sample) have been produced in 20-40 seconds (Figure 7).

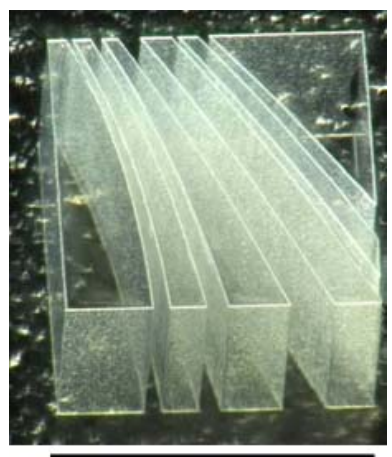

$1 \mathrm{~mm}$

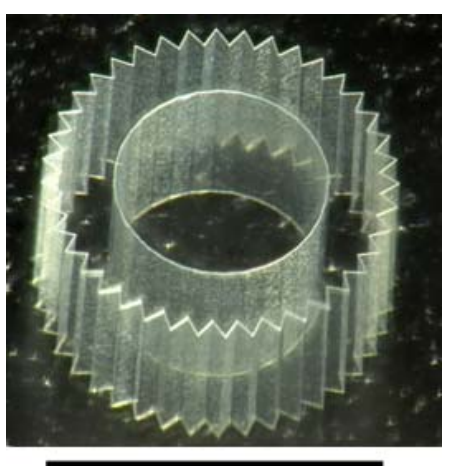

$1 \mathrm{~mm}$
Figure 7: Micro parts in $1 \mathrm{~mm}$ sapphire

Using subsequent wet chemical etching e.g. in aqueous solution of hydrofluoric acid the modified volume can be removed and the part as well as the shaped hole may be obtained [9]. Further development of the processing strategy is necessary to prevent the formation of cracks during the in-volume selective laser etching of complex 3D shaped parts and to prevent the formation of periodical nanostructures (coherent in-volume sub-wavelength ripples, nanoplanes with a period of 340-350 nm [10]), which would prevent the releasing of the etched part.

\subsection{Periodical nanostructures}

Micro markings on the surface of sapphire are produced by direct laser writing using a numerical aperture of $\mathrm{NA}=0.6$. Using a repetition rate of $500 \mathrm{kHz}$, an average power of $350 \mathrm{~mW}$ an a scanning velocity of $50 \mathrm{~mm} / \mathrm{s}$ a picture is written in 15 seconds (Figure 8, top). Using scanning electron microscopy after cleaning the sample in an ultrasonic bath periodical nanostructures (coherently continued sub-wavelength ripples) are observed in the laser written lines with a period of $340 \mathrm{~nm}$, which is the same period as observed in the volume of sapphire during involume selective laser etching and which is also obtained using writing velocities of $1 \mathrm{~mm} / \mathrm{s}$ or less using the same repetition rate [10]. At intersections of perpendicular lines and at sharp turns a loss of coherence is observed while in rounded curves and straight lines the sub-wavelength ripples are coherently continued (Figure 8 , bottom).

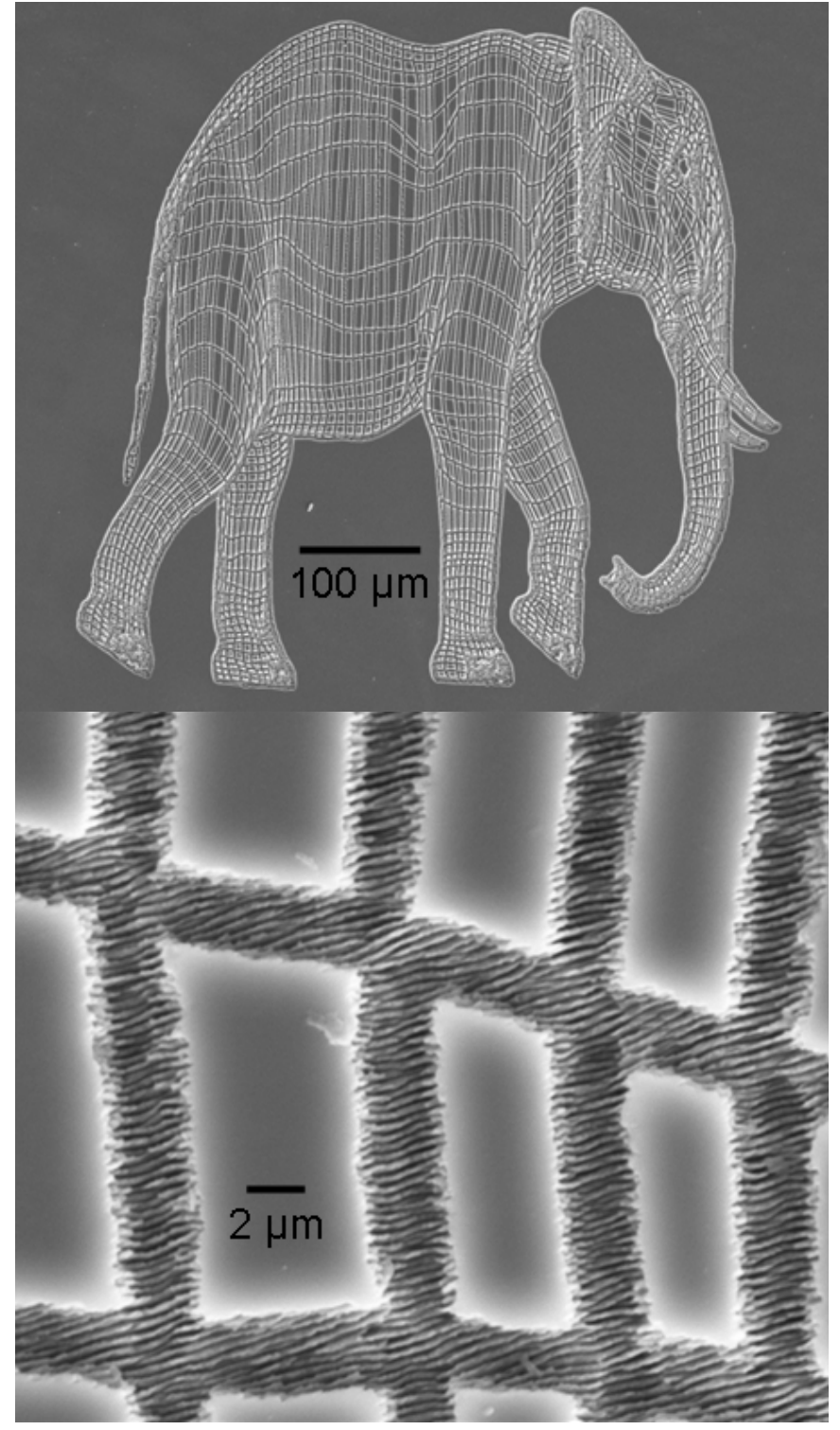

Figure 8: Periodical nanostructures by high speed writing in the surface of sapphire: Structure (top) and detail with coherent sub-wavelength ripples (bottom)

\section{Conclusions}

A scanning system consisting of commercial galvo mirrors, imaging optics, microscope objective and computer controlled pre-compensation of spherical aberrations has been build to enable high speed micro structuring in the volume of transparent materials. A numerical aperture of $0.4-1.3$ results in small focal volume $\left(<1 \mu \mathrm{m}^{3}\right)$, high precision (up to less than $100 \mathrm{~nm}$ ), high writing velocities (up to $360 \mathrm{~mm} / \mathrm{s}$ ) and high dynamics. Using existing CAD/CAM software allows for rapid manufacturing of micro structured parts, waveguides, in-volume micro markings and nano structures fully exploiting the potential productivity of today's high repetition rate FCPA fs-lasers.

Straight and curved waveguides have been written in boro-silicate glass using scanning velocities of 50-100 $\mathrm{mm} / \mathrm{s}$. Micro markings in the volume of fused silica have been produced in less than 1 second. Crack-free modifications inside sapphire have been produced and a colored marking has been written in 3 seconds exploiting diffrac- 
tion and interference in the observers' eye. 3D structures have been produced in sapphire using in-volume selective laser etching and coherently continued sub-wavelength ripples have been produced with scanning velocity of 50 $\mathrm{mm} / \mathrm{s}$ exhibiting the same period of $340 \mathrm{~nm}$ as obtained by much slower scanning previously.

Further work will include the development of processing strategies for complex shaped $3 \mathrm{D}$ micro parts. The transfer of the process as well as the scanning system to industrial applications is the next step to increase the productivity and precision exploiting today's reliable efficient fs-lasers like FCPA lasers.

\section{Acknowledgments and Appendixes}

(1) We would like to thank IMRA America for the possibility to use the $\mu$ Jewel laser and the acousto-optical modulator in the framework of the Premier Application Lab at LLT. (2) We are grateful to Fraunhofer ILT for founding the hardware used to build the scanning system.

\section{References}

[1] K.M. Davis, K. Miura, N. Sugiomto, K. Hirao: Writing waveguides in glass with a femtosecond laser, Optics Letters, 21, (1996), p. 1729

[2] C. Hnatovsky, R.S. Taylor, E. Simova, P.R. Rajeev, D.M. Rayner, V.R. Bhardwaj, P.B. Corkum: Fabrication of microchannels in glass using focused femtosecond laser radiation and selective chemical etching, Appl. Phys. A 84, (2006), p. 47
[3] Y. Bellouard, A. Said, M. Dugan, P. Bado: Fabrication of high-aspect ratio micro fluidic channels and tunnels using femtosecond laser pulses and chemical etching, Optics Express 12, (2004), p. 2120

[4] K. Sugioka, Y. Hanada, K. Midorikawa: 3D Microstructuring of glass by femtosecond laser direct writing and application to biophotonic microchips, Progress in Electromagnetics Research Letters 1, (2008), p. 181.

[5] S.M. Eaton, H. Zhang, P.R. Herman: Heat accumulation effects in femtosecond laserwritten waveguides with variable repetition rate. Optical Society of America $13,(2005)$, p. 4708-4716.

[6] C. B. A. Schaffer, A. Brodeur, E. Mazur: Measurement Science and Technology, 12, (2001), p. 1784.

[7] United States Patent No. 4953 962, (1990)

[8] D. Esser, D. Mahlmann, D. Wortmann, J. Gottmann: Interference microscopy of femtosecond laser written waveguides in phosphate glass, Appl. Phys. B 96, (2009) p. 453.

[9] M. Hörstmann-Jungemann, J. Gottmann, M. Keggenhoff: 3D micro structuring of sapphire using fs-laser irradiation and selective etching, Submitted for publication in this journal.

[10] J. Gottmann, D. Wortmann, M. HörstmannJungemann: Fabrication of sub-wavelength surface ripples and in-volume nanostructures by fs-laser induced selective etching, Applied Surface Science, 255, (2009), p. 5641 